
internationalen Fachliteratur referiert und kommentiert.

\section{Ist die Adenotonsillektomie wirksam bei Schlafapnoen im Kindesalter?}

Die Adenotonsillektomie wird bei Kindern mit obstruktiver Schlafapnoe häufig durchgeführt. Ihr Nutzen hinsichtlich der Verminderung von Symptomen, der Verbesserung von Schulleistungen und einer besseren Lebensqualität ist jedoch bislang nicht eingehend untersucht worden. Eine Arbeitsgruppe um Susan Redline in Boston, Massachusetts, USA, wollte herausfinden, ob die Adenotonsillektomie für Kinder mit obstruktiver Schlafapnoe in der Altersgruppe von fünf bis neun Jahren wirklich vorteilhafter ist als aufmerksames Beobachten und Abwarten für die nächsten sieben Monate.

$\mathrm{D}$ ie Autoren haben 464 Kinder in der genannten Altersgruppe randomisiert in eine Gruppe, bei der eine frühzeitige Adenotonsillektomie durchgeführt wurde, und eine Kontrollgruppe, bei der abgewartet wurde. Es wurden polysomnographische Untersuchungen, kognitive Tests und Verhaltenstests durchgeführt und der Gesundheitszustand bei Eintritt in die Studie und nach sieben Monaten überprüft.

Die Lehrer der Kinder waren für die Beurteilung des Verhaltens miteinbezogen worden, sie füllten strukturierte Fragebögen aus. Den Eltern wurden zur Evaluierung schlafbezogener Atemstörung ebenfalls strukturierte Fragebögen zur Verfügung gestellt.

Etwa die Hälfte der Teilnehmer war übergewichtig oder adipös. In den neuropsychologischen Tests entsprachen die Untersuchungsergebnisse zu Beginn der Studie dem Bevölkerungsdurchschnitt.

Die ausgewerteten Daten zeigten, dass die frühe Adenotonsillektomie nicht zu einer Verbesserung in den neuropsychologischen Testergebnissen geführt hatten. Jedoch waren in der Interventionsgruppe signifikante Verbesserungen im Verhalten, in der Lebensqualität und bei den polysomnographischen Befunden zu verzeichnen. Bei $79 \%$ der operativ behandelten Kinder kam es zur Normalisierung der polysomnographischen Befunde, allerdings war dies auch bei $46 \%$ der nicht operierten Kinder der Fall.

Fazit: Es ist wenig überraschend, dass sich die Ergebnisse der neuropsychologischen Tests durch die frühe Operation nicht wirklich verbessern. Man hätte ja sonst erwarten müssen, dass schon im Ausgangsbefund die Kinder mit obstruktiver Schlafapnoe schlechter abschneiden als der Durchschnitt der Bevölkerung. Immerhin verdient festgehalten zu werden, dass sich die polysomnographischen Befunde signifikant besserten und auch die sekundären Outcomeparameter wie Verhalten und Lebensqualität verbessert wurden. Es kann daher das Fazit gezogen werden, dass die Adenotonsillektomie bei Schlafapnoen in der Altersgruppe von fünf bis neun Jahren eine sinnvolle Maßnahme ist. Man darf aber nicht erwarten, dass sich die Schulleistungen der Kinder dadurch unmittelbar verbessern.

Dr. Hartmut Koch

Marcus CL et al. A randomized trial of adenotonsillectomy for childhood sleep apnea.

N Engl J Med 2013; 368: 2366-76

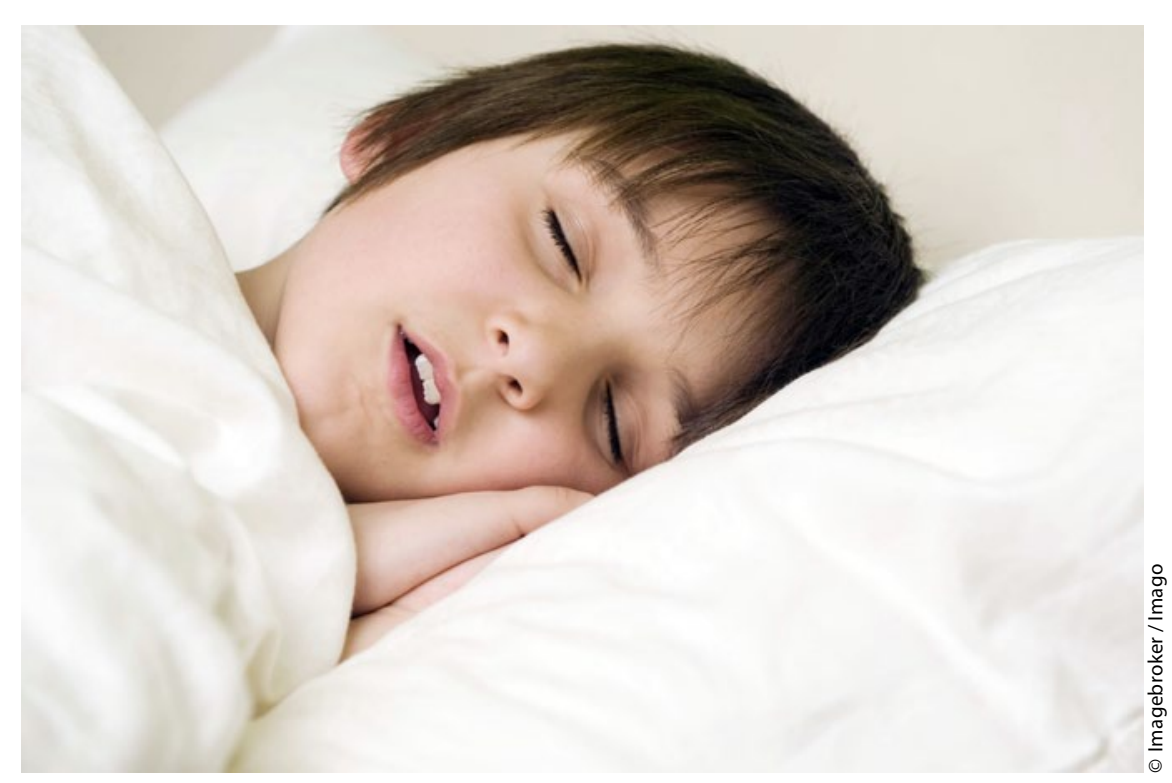

Schätzungen zufolge leiden zwischen ein und zwei Prozent aller Kinder an obstruktiver Schlafapnoe. Bei Kindern mit ADHS ist es sogar jedes vierte Kind. 